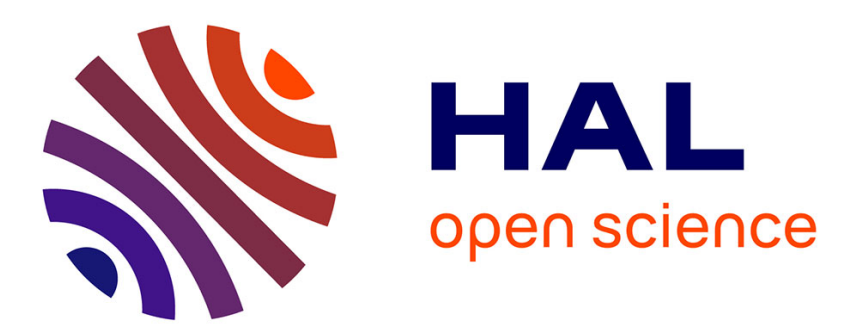

\title{
Non-volatile electric control of spin-charge conversion in a SrTiO3 Rashba system
}

Paul Noël, Felix Trier, Luis M. Vicente Arche, Julien Bréhin, Diogo Vaz, Vincent Garcia, Stéphane Fusil, Agnès Barthélémy, Laurent Vila, Manuel Bibes, et al.

\section{To cite this version:}

Paul Noël, Felix Trier, Luis M. Vicente Arche, Julien Bréhin, Diogo Vaz, et al.. Non-volatile electric control of spin-charge conversion in a SrTiO3 Rashba system. Nature, 2020, 580 (7804), pp.483-486. 10.1038/s41586-020-2197-9 . hal-02552857

\section{HAL Id: hal-02552857 https://hal.science/hal-02552857}

Submitted on 24 Apr 2020

HAL is a multi-disciplinary open access archive for the deposit and dissemination of scientific research documents, whether they are published or not. The documents may come from teaching and research institutions in France or abroad, or from public or private research centers.
L'archive ouverte pluridisciplinaire HAL, est destinée au dépôt et à la diffusion de documents scientifiques de niveau recherche, publiés ou non, émanant des établissements d'enseignement et de recherche français ou étrangers, des laboratoires publics ou privés. 


\title{
Non-volatile electric control of spin-charge conversion using a $\mathrm{SrTiO}_{3}$ Rashba system
}

\author{
Paul Noël ${ }^{1 * \star}$, Felix Trier $^{2 *}$, Luis M. Vicente Arche ${ }^{2}$, Julien Bréhin ${ }^{2}$, Diogo C. Vaz ${ }^{2 \bullet}$, Vincent \\ Garcia $^{2}$, Stéphane Fusil ${ }^{2,3}$, Agnès Barthélémy ${ }^{2}$, Laurent Vila ${ }^{1}$, Manuel Bibes ${ }^{2}$ and Jean-Philippe \\ Attané ${ }^{* *}$ \\ ${ }^{1}$ Univ. Grenoble Alpes, CEA, CNRS, Grenoble INP, Spintec, 38000 Grenoble, France \\ 2 Unité Mixte de Physique, CNRS, Thales, Université Paris-Saclay, 91767 Palaiseau, France \\ 3 Université d’Evry, Université Paris-Saclay, Evry, France
}

After $\mathbf{5 0}$ years of exponential increase in computing efficiency, the technology of today's electronics is approaching its physical limits, with feature sizes smaller than $10 \mathrm{~nm}$. New schemes must be devised to contain the ever-increasing power consumption of information and communication systems ${ }^{1}$, which requires the introduction of non-traditional materials and new state variables. As recently highlighted ${ }^{2}$, the remanence associated with collective switching in ferroic systems is appealing to reduce power consumption. A particularly promising approach is spintronics, which relies on ferromagnets to provide non-volatility and to generate and detect spin currents ${ }^{3}$. However, magnetization reversal by spin transfer torques ${ }^{4}$ is a power consuming process. This is driving research on multiferroics to achieve a low-power electricfield control of magnetization ${ }^{5}$, but practical materials are scarce and magnetoelectric switching remains difficult to control. Here, we demonstrate an alternative strategy to achieve low-power spin detection, in a non-magnetic system. We harness the electric-field-induced ferroelectriclike state of $\mathrm{SrTiO}_{3}{ }^{6-9}$ to manipulate the spin-orbit properties ${ }^{10}$ of a two-dimensional electron gas $^{11}$, and efficiently convert spin currents into positive or negative charge currents, depending on the polarisation direction. This non-volatile effect opens the way to the electric-field control of spin currents and to ultralow-power spintronics, in which non-volatility would be provided by ferroelectricity rather than by ferromagnetism.

\footnotetext{
* these authors contributed equally to this work.

- Now at CIC Nanogune, Tolosa Hiribidea 76, 20018 Donostia - San Sebastian,Spain.

^Now at ETH Zürich, Hönggerbergring 64, CH-8093 Zürich, Switzerland

- manuel.bibes@cnrs-thales.fr * jean-philippe.attane@cea.fr
} 
Spin-orbitronics ${ }^{12}$ exploits the interplay between charge and spin currents enabled by the spin-orbit coupling (SOC) in non-magnetic systems. It allows the generation of pure spin currents from charge currents and vice-versa, without resorting to ferromagnetic materials. The Edelstein effect ${ }^{13}$ allows charge-spin conversion ${ }^{14}$ with an efficiency comparable to or larger than that of

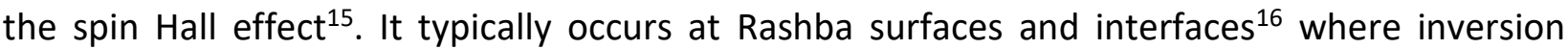
symmetry breaking results in an out-of-plane electric field. In the presence of SOC, this leads to a locking of the momentum and spin degrees of freedom. The flow of an in-plane charge current in such a system produces a transverse spin density, which can diffuse as a spin current in an adjacent material ${ }^{13}$. Conversely, injecting a spin density results in the production of a net charge current by inverse Edelstein effect ${ }^{17}$. As such, Rashba systems can be used as spin generators and detectors, but with an efficiency inherently set by the electronic structure, without the possibility to switch it by an external stimulus.

In comparison with ferromagnets, the order parameter of ferroelectrics (polarisation) can be switched by an electric field for energy costs typically 1000 times smaller ${ }^{2}$. Moreover, ferroelectrics can harbour intense electric fields, largely modifying the carrier densities in adjacent materials, and thereby tuning their properties in a non-volatile fashion. An exciting route towards low power electronics would thus be to combine the remanence of ferroelectrics with the ability to generate and manipulate spin currents by the direct and inverse Edelstein effects in Rashba systems. Beyond magnetoelectricity, ferroelectric Rashba architectures would therefore offer a new approach for the non-volatile control of spin currents by electric fields, with an ultralow-power operation.

Most efforts to identify single-phase Rashba ferroelectrics ${ }^{18}$ have focused on $\mathrm{GeTe}^{19}$. However, because of high leakage ferroelectric properties are poor ${ }^{20}$ and spin-charge conversion experiments have yielded a moderate efficiency ${ }^{21}$. Here we show that beyond bulk materials, interface systems combining Rashba SOC and a switchable polarisation enable the non-volatile electrical control of a highly efficient spin-charge conversion.

The general concept of ferroelectrically-controlled spin-charge conversion is described in Fig. 1. At the interface between a ferroelectric and an ultrathin SOC system (a heavy metal, a Weyl semi-metal, a two-dimensional electron gas - 2DEG -, etc.), electrons are accumulated or 
depleted depending on the polarisation direction (Fig. 1a). This modifies the electric field in the interface region, and in the ideal case changes its sign. If a Rashba state is present in the SOC system at the interface with the ferroelectric, reversing the sign of the local electric field reverses the chirality of the spin textures in both split Fermi contours (Fig. 1b). Through the inverse Edelstein effect ${ }^{13}$, the injection of a spin current into the Rashba state will produce a charge current $J_{c}$ whose sign will depend on the ferroelectric polarisation state (Fig. $1 \mathrm{c}$ ). This mechanism offers the possibility to design a wealth of devices such as the bipolar memory proposed in Fig. 1. It can also be the basis of logic devices ${ }^{22}$ akin to the magnetoelectric spin-orbit (MESO) device proposed by Intel ${ }^{23}$, but without resorting to a multiferroic to switch the ferromagnet.

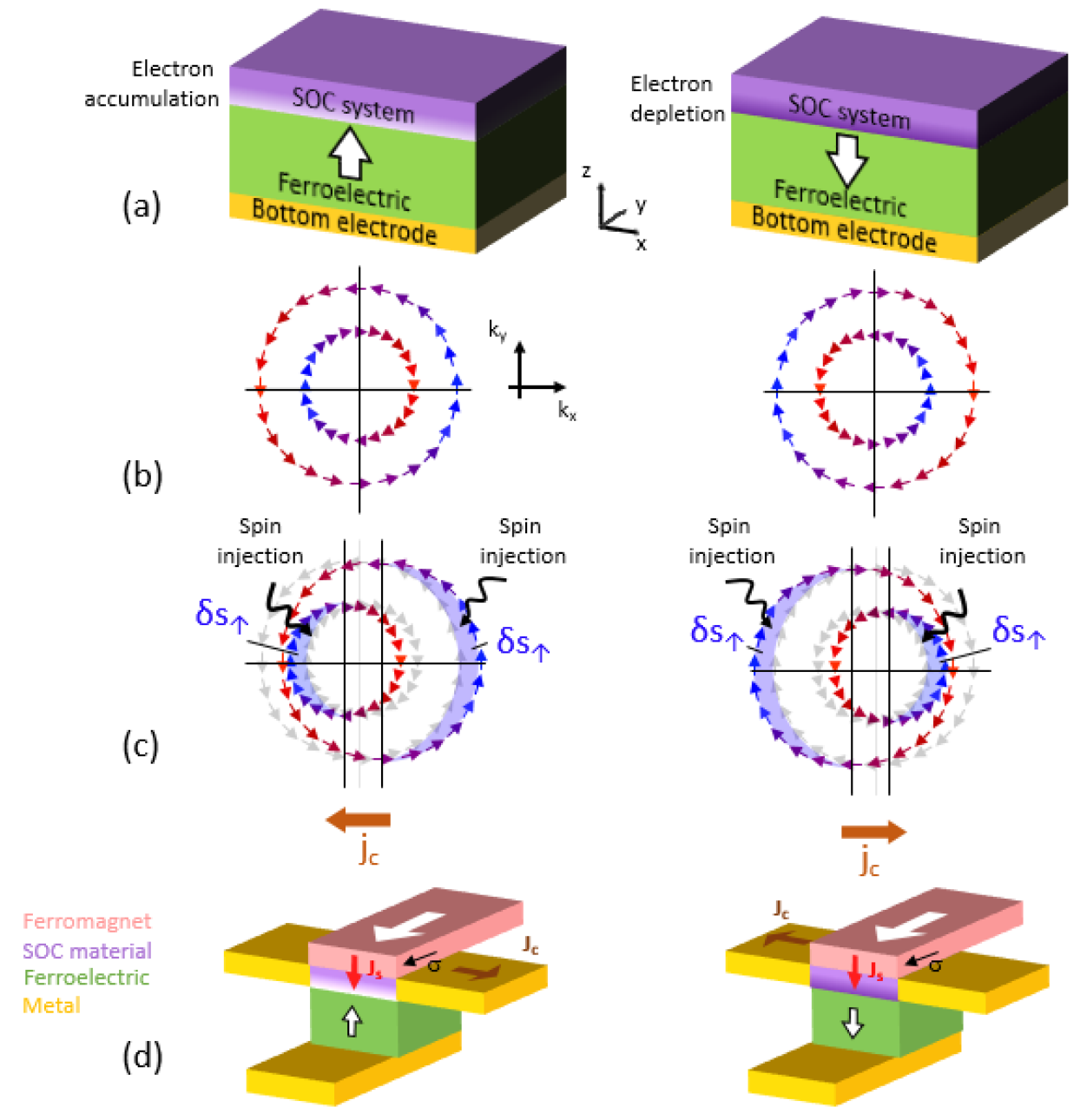

Fig.1. Concept of ferroelectrically-controlled spin-charge conversion. (a) Sketch of a ferroelectric Rashba architecture combining a ferroelectric material (green) and a material with spin-orbit coupling (purple). Upon switching polarisation, electrons are accumulated (left) or depleted (right) 
in the SOC material (e.g. a 2DEG), creating an electric field whose sign depends on the polarisation direction. (b) Corresponding Rashba-split chiral Fermi contours with spin-momentum locking. The chirality of the contours switches upon switching the ferroelectric polarisation. (c) Inverse Edelstein effect in a Rashba interface. When a spin current is injected (e.g. by spin pumping) with a spin polarisation along the $y$ axis, the spin population is altered, causing a displacement of the two inequivalent Fermi surfaces (red and blue lines) by $\pm \Delta k$ in momentum space. This results in $a$ net charge current generated perpendicularly to the spin current and to its spin polarisation. The sign of the generated current depends on the chirality of the Fermi contours and is thus reversed upon switching ferroelectric polarisation. (d) Non-volatile device operated by ferroelectricity and Rashba SOC. Through the inverse Edelstein effect a charge current $J_{C}$ is generated by the conversion of a spin current $J_{S}$ injected from the ferromagnet. The sign of $J_{C}$ changes with the direction of the ferroelectric polarisation.

To experimentally demonstrate the non-volatile electrical control of the spin-charge conversion, we use $\mathrm{SrTiO}_{3}$ (STO) 2DEGs, generated by the deposition of a film of Al onto a STO single crystal ${ }^{24,25}$. Indeed, STO 2DEGs exhibit a sizeable Rashba SOC ${ }^{10}$ with a very high conversion efficiency ${ }^{25,26}$. In addition, STO is a quantum paraelectric that develops an electric-field-induced switchable polarisation at low temperature ${ }^{7-9}$.

The spin-to-charge conversion was measured using spin pumping by ferromagnetic resonance on a $\mathrm{NiFe}(20 \mathrm{~nm}) / \mathrm{Al}(0.9 \mathrm{~nm}) / / \mathrm{STO}$ sample (cf. sketch in the inset of Fig. 2a). The nominally $500 \mu \mathrm{m}$ thick $\mathrm{SrTiO}_{3}$ (STO) substrate was thinned down to $250 \pm 20 \mu \mathrm{m}$ using mechanical polishing, allowing the application of high electric fields $(E)$. A static magnetic field was applied along the $y$ direction. At the ferromagnetic resonance, a pure spin current is injected into the $2 D E G$ along the $-z$ direction, with spins oriented along $y^{27}$. The measurement of the extra damping due to this relaxation channel allows calculating the injected spin current ${ }^{26,27}$. In the $2 \mathrm{DEG}$, this spin current is then converted into a charge current oriented along $\mathrm{x}$ by the inverse Edelstein effect. Since the sample is in open circuit, at the resonance field this results in a voltage drop along the sample, in the $x$ direction ${ }^{26}$. 
In the pristine, ungated state, the voltage drop obtained at resonance corresponds to the production of a positive normalized current of $1.2 \mathrm{~A} \cdot \mathrm{mT}^{-2} \cdot \mathrm{m}^{-1}$ (top left panel of Fig. $2 \mathrm{~b}$ ). At low temperature, STO is known to undergo a phase transition at high electric field ${ }^{7-9}$ : Once a large electric field has been applied, the material develops a switchable, remanent polarisation. This phenomenon is often referred to as a field-induced ferroelectric order or a field-induced ferroelectric-like state. We applied voltages up to $\pm 200 \mathrm{~V}$, corresponding to $E$ up to $\pm 8 \mathrm{kV} / \mathrm{cm}$, high enough to achieve this phase transition ${ }^{7,9}$. After a first initialization cycle [+200 V; $-200 \mathrm{~V}$; $+200 \mathrm{~V}]$, the gate voltage dependence of the spin pumping signal shows a hysteretic behaviour (Fig. 2a). The charge currents produced at ferromagnetic resonance have opposite signs for +200 $\mathrm{V}$ and $-200 \mathrm{~V}$ gate voltages, as seen in points $\mathrm{B}, \mathrm{F}$ and $\mathrm{D}$ of Fig. $2 \mathrm{a}$ and $2 \mathrm{~b}$. After applying the maximum voltage, the normalized current reaches a very high amplitude $\left( \pm 8.8 \mathrm{~A} \cdot \mathrm{mT}^{-2} \cdot \mathrm{m}^{-1}\right)$, beyond the record values obtained previously in LAO/STO and Al/STO samples (around 5 A.mT ${ }^{-}$ $\left.{ }^{2} \cdot \mathrm{m}^{-1}\right)^{26}$. The spin-charge conversion efficiency is quantified by the inverse Edelstein length $\lambda_{\text {IEE, }}$ equal to the ratio of the produced 2D charge current density by the injected 3D spin current, i.e., $\lambda_{I E E}=j_{\mathrm{c}}^{2 \mathrm{D}} / j_{\mathrm{s}}^{3 \mathrm{D}}\left(\right.$ Ref. $\left.{ }^{17}\right)$, cf. Methods. Here, we estimate $\lambda_{\mathrm{IEE}} \approx \pm 60 \mathrm{~nm}$, a value one to two orders of magnitude larger than in metallic Rashba interfaces ${ }^{17}$ or topological insulators ${ }^{28}$.

Remarkably, the produced current - and thus the spin-charge conversion rate - is remanent at $\mathrm{V}_{\text {gate }}=0 \mathrm{~V}$, as seen in $\mathrm{C}$ and $\mathrm{E}$. Similar hysteretic behaviors have been obtained on several thinned-down samples but not on a $500 \mu \mathrm{m}$-thick STO substrate, which indicates the existence of a critical electric field for the hysteresis to appear. The non-volatile control of the spin-charge conversion is further evidenced by Fig. 2c, which displays the produced normalized charge current at $0 \mathrm{~V}$ after the application of $500 \mathrm{~ms}$ pulses of $\pm 200 \mathrm{~V}$ gate voltage. Fig. $2 \mathrm{~d}$ shows the temperature dependence of the difference $\Delta \mathrm{I}_{\mathrm{C}}$ in the produced current obtained at remanence after applying pulses of $+200 \mathrm{~V}$ and $-200 \mathrm{~V}$ at $7 \mathrm{~K} . \Delta \mathrm{l} \mathrm{c}$ is large below $30 \mathrm{~K}$ and vanishes above $45-50 \mathrm{~K}$, suggesting a transition of STO into the paraelectric phase ${ }^{7-9}$. Extended Data Figures 1 and 4 show that the effect is reproducible and stable in time for at least several hours. 

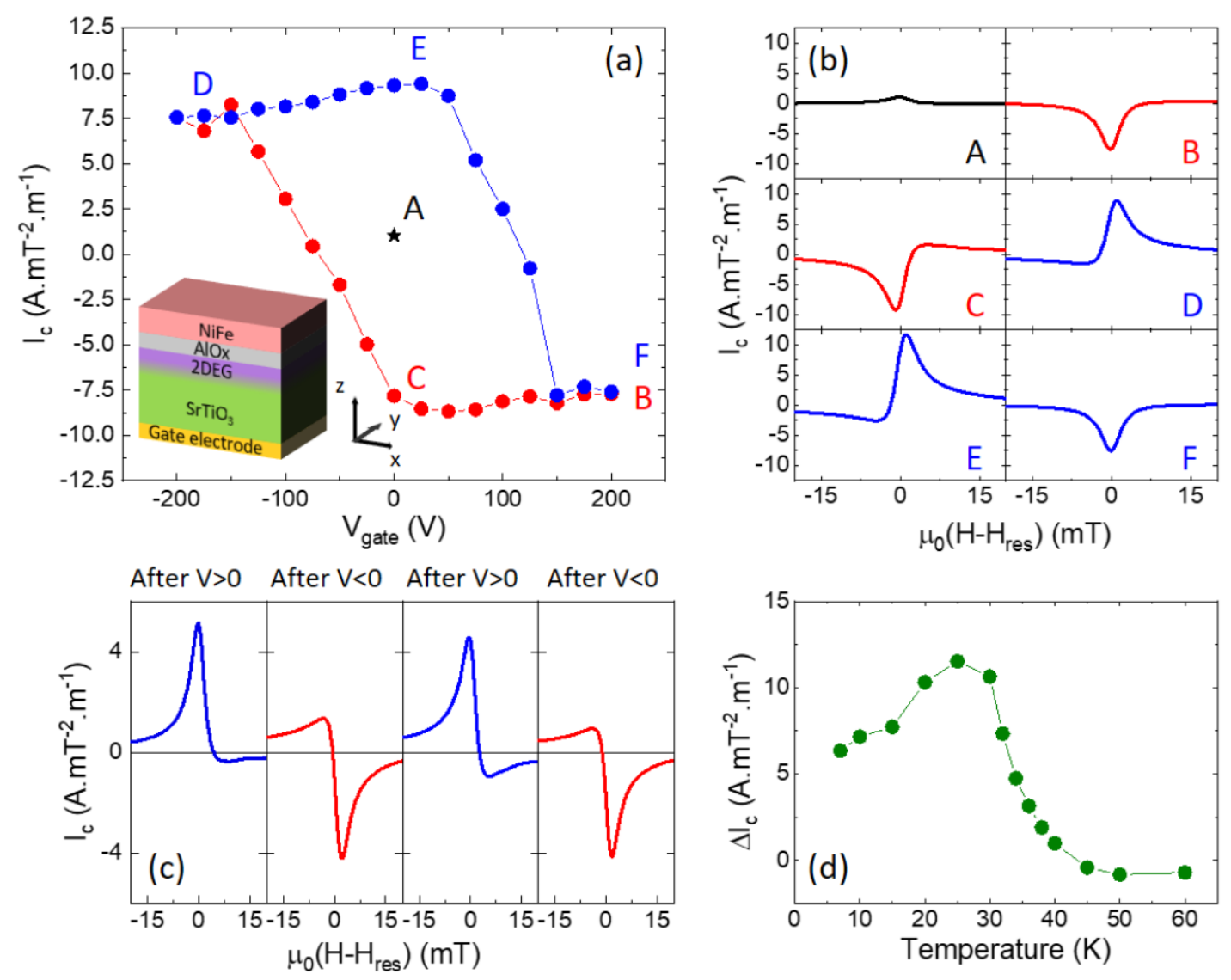

Fig. 2. Electric-field controlled spin-charge conversion with electrical remanence. (a) Gate voltage dependence of the normalized current produced by the inverse Edelstein effect. The inset shows a sketch of the heterostructure. (b) Dependence of the normalized current produced with the magnetic field in spin pumping experiments, for different voltage values (cf. panel (a)). (c) Produced normalized charge current at electrical remanence after applying positive or negative voltage pulses of $\pm 200 \mathrm{~V}$. All data have been measured at $7 \mathrm{~K}$. (d) Temperature dependence of the difference between the remanent normalized currents after the application of a large positive or negative voltage.

We have also performed electric polarisation measurements on a $\mathrm{Al}(1.8 \mathrm{~nm}) / / \mathrm{STO} 2 \mathrm{DEG}$ sample with a STO thickness of $200 \pm 20 \mu \mathrm{m}$. As visible in Fig. 3a, the application of an electric field up to $2.5 \mathrm{kV} / \mathrm{cm}$ (green curve) yields a linear dependence of the polarisation with $E$, as expected for a dielectric. However, when the voltage exceeds $\sim 7 \mathrm{kV} / \mathrm{cm}$, a hysteresis develops, associated with switching current peaks in the I vs $E$ data (Fig. 3a, inset). The saturation polarisation is about 
$4 \mu \mathrm{C} / \mathrm{cm}^{2}$, in agreement with earlier reports ${ }^{7}$. Upon increasing the temperature (Fig. 3c), the loop progressively closes, indicating a Curie temperature close to $50 \mathrm{~K}$ (Fig. 3d). This almost coincides with the temperature at which the remanent spin-charge conversion effect vanishes (Fig. 2d), strongly suggesting that the switchable polarisation is at the origin of the hysteretic inverse Edelstein effect. At low temperature, reducing $E$ to below the critical value still yields hysteretic polarisation loops, albeit with a lower remanent polarisation (Fig. 4a).
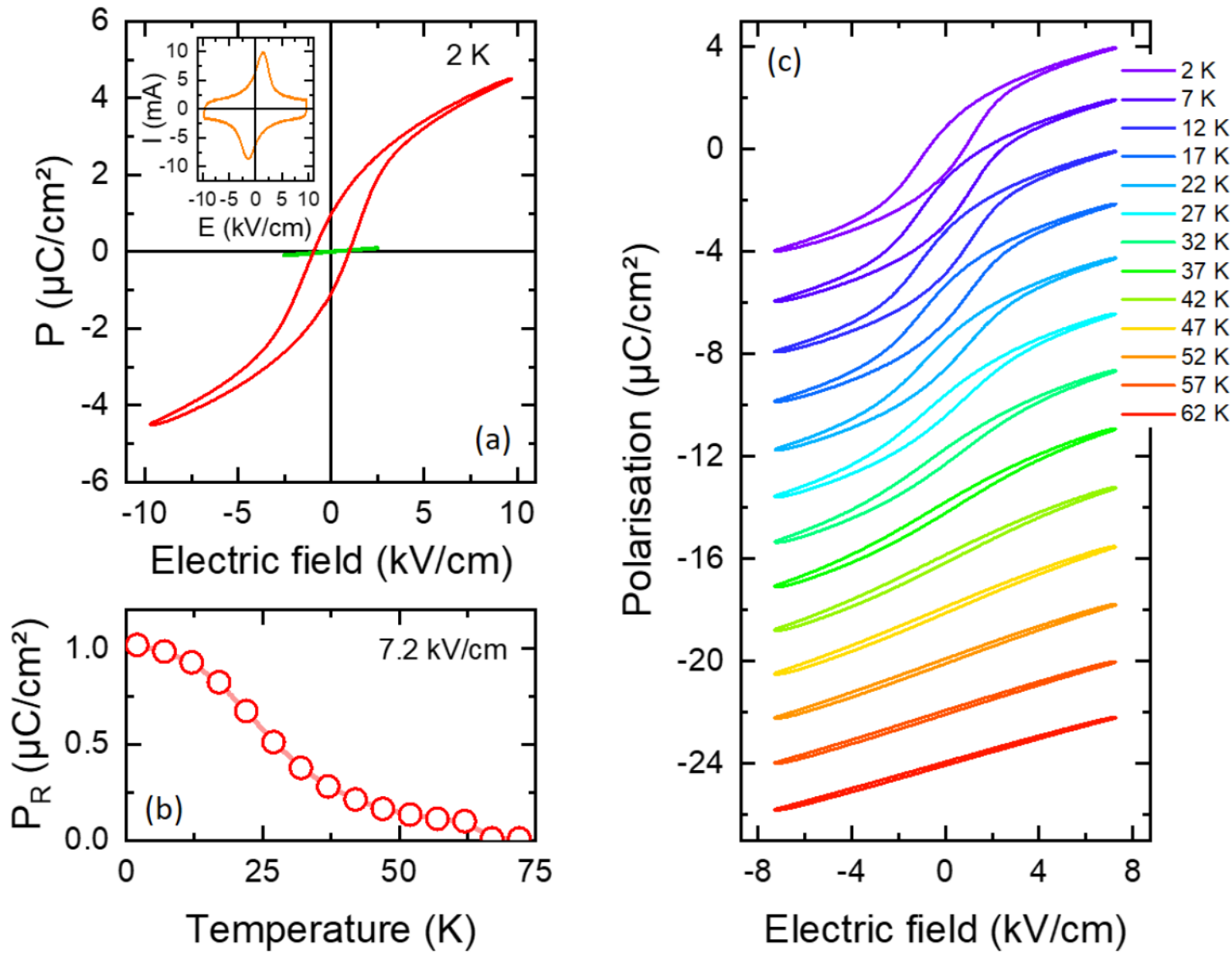

Fig. 3. Electric polarisation measurements. (a) Polarisation vs voltage curves measured on a Al(1.8 nm)//STO sample. The green curve corresponds to the polarisation loop measured with a maximum field of $2 \mathrm{kV} / \mathrm{cm}$. Inset: Corresponding current vs voltage curve. (b) Temperature dependence of the remanent polarisation $P_{R}$. (c) Polarisation loops at different temperatures. 
One of the hallmark features of STO 2DEGs is the strong gate voltage dependence of the sheet resistance $R_{s}{ }^{29}$. In thick STO samples the gate dependence of $R_{s}$ is usually non hysteretic ${ }^{30}$, in line with the paraelectric nature of STO at low electric fields. Here, as seen in Fig. $4 \mathrm{~b}, R_{s}$ varies as the carrier density varies, but this dependence also exhibits a clear hysteresis, allowing the non-volatile electrical control of the 2DEG electronic properties. Remarkably, the hysteresis amplitude increases upon increasing the maximum $E$, so that the $R_{S}$ vs. $E$ loops mimic the polarisation loops of Fig. 4a. Hall measurements made in the two remanent states yield a difference in carrier densities $\Delta n_{s}=5.45 \times 10^{12} \mathrm{~cm}^{-2}$, only two times smaller than the theoretical value $\Delta n_{s}=2 P_{r} / e=1.13 \times 10^{13} \mathrm{~cm}^{-2}$ (using $P_{r}=0.9 \mu \mathrm{C} / \mathrm{cm}^{2}$ ), thus corresponding to a remarkable efficiency compared to the literature ${ }^{31,32}$. Note that we have also performed $R_{s} v s$. $E$ loops on spinpumping samples, which possess a NiFe layer, showing that the obtained loops are very similar to the $J_{C}$ vs. $E$ loops (cf. Extended Data Figure 1 and Methods).
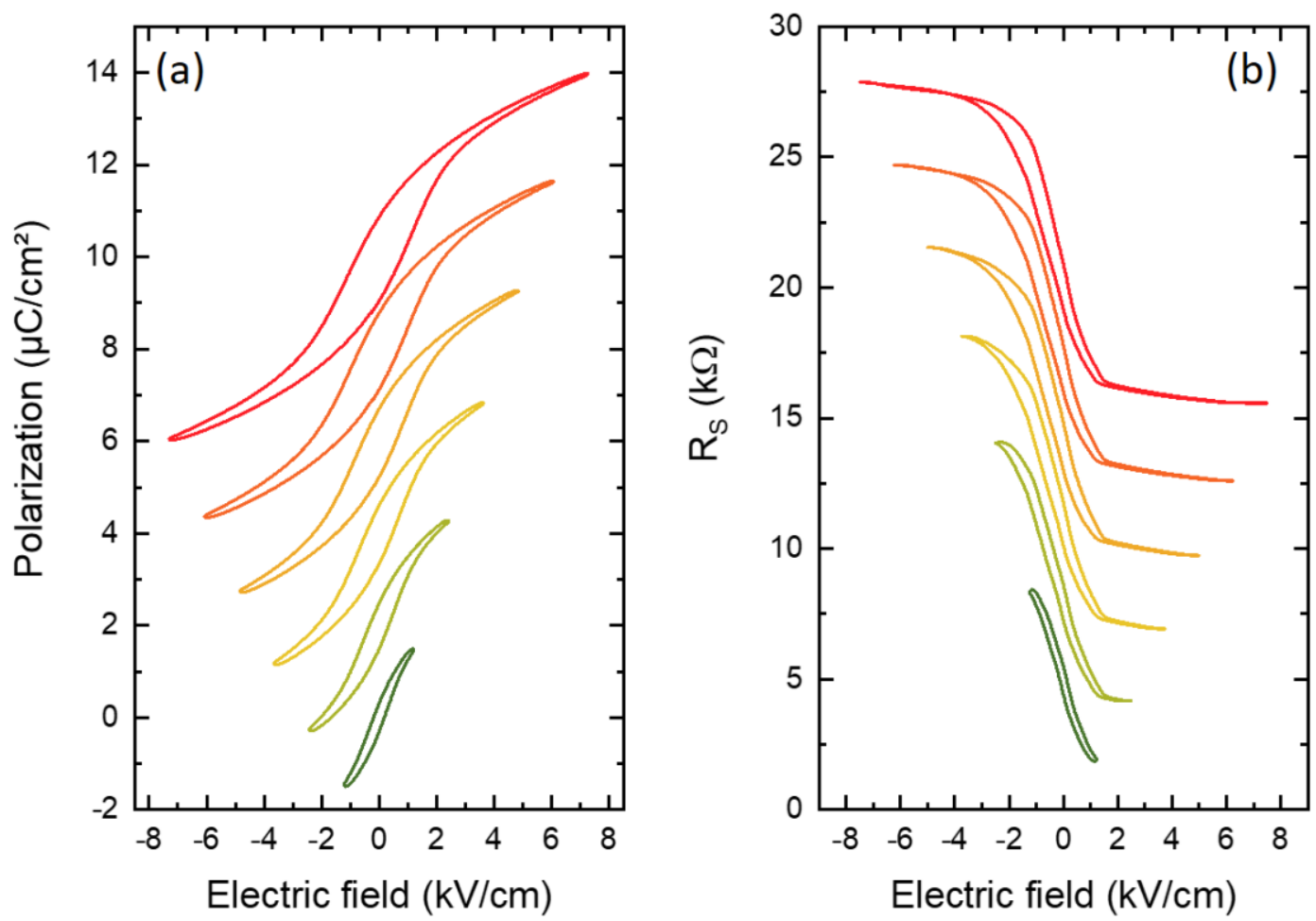

Fig. 4. Field effect experiments. (a) Polarisation loops at $7 \mathrm{~K}$ measured in the field-induced state for different increasing maximum electric fields. The curves are shifted by $2 \mu \mathrm{C} / \mathrm{cm}^{2}$ for clarity. (b) Gate dependence of the 2DEG sheet resistance for different maximum electric fields at $2 \mathrm{~K}$. The curves are shifted by $3 \mathrm{k} \Omega$ for clarity. 
Several mechanisms may be invoked to explain our observation of a hysteretic inverse Edelstein effect. One can be related to the description of Fig. 1a, namely a local inversion of the electric field in the SOC material (here the 2DEG) promoting polarisation-direction-dependent Rashba SOC and spin-charge conversion. Additionally, electronic structure effects may be at play, since the multiorbital band structure of STO 2DEGs is known to produce effective Rashba effects with opposite signs, depending on the orbitals involved ${ }^{25,26}$. Moreover, the presence of a switchable polarisation with associated polar displacements of cations and anions should significantly modify the band structure compared to the paraelectric case. This may generate additional (avoided) band crossings, possibly with non-trivial topology ${ }^{25}$, leading to super-efficient spin-charge conversion.

Our results constitute the basis of a new type of spintronics, in which non-volatility would not originate from ferromagnetism but from ferroelectricity. They could be extended to room temperature by designing 2DEGs on strained STO thin films ${ }^{33}$ or $\mathrm{BaTiO}_{3}{ }^{24}$ for instance. This could open the way to a whole new class of ultralow-power spin-orbitronic devices (memories, spin field-effect transistors, spin Hall transistors or MESO-like logic devices). In the future, the demonstration of a non-volatile electric control of the direct Edelstein effect could additionally lead to reconfigurable spin-orbit torque memories and logic gates, benefit to skyrmions or domain walls manipulation, and allow the development of agile $\mathrm{THz}$ emitters and spin-wave logic architectures.

\section{Methods}

Sample preparation. NiFe and Al films were deposited at room temperature by dc magnetron sputtering on $\mathrm{TiO}_{2}$-terminated (001)-oriented STO substrates (from CrysTec $\mathrm{GmbH}$ ). $\mathrm{TiO}_{2}$ termination was achieved through a chemical treatment, where the substrate was submerged in a buffered hydrofluoric acid ( $\left.\mathrm{NH}_{4} \mathrm{~F}-\mathrm{HF} 7: 1\right)$ for $30 \mathrm{~s}$ and annealed under a rich oxygen environment at $1000^{\circ} \mathrm{C}$ for $3 \mathrm{~h}$. Before deposition, the STO substrates were additionally annealed at $730{ }^{\circ} \mathrm{C}$ for $2 \mathrm{~h}$ under a partial oxygen pressure of 400 mbar. The deposition of the metallic layers was 
performed under an Ar partial pressure of $4.5 \times 10^{-4}$ mbar and a substrate-to-target distance of $7 \mathrm{~cm}$. The samples including NiFe were additionally capped with a $2.5 \mathrm{~nm}$ layer of Al, which becomes oxidized when exposed to air. Samples were mechanically polished on diamond pads under deionized water flow.

Spin pumping. The spin-pumping experiments were carried out using a Bruker ESP300E X-band CW spectrometer at $9.68 \mathrm{GHz}$, with a loop-gap Bruker ER 4118X-MS5 cavity, and using a microwave power of $5 \mathrm{~mW}$ or less to remain in the linear regime. The generated DC voltage was measured using a Keithley 2182A nanovoltmeter. The gate voltage was applied using a Keithley 2400 sourcemeter. The measured signals were observed to be linear with the rf power up to 5 $\mathrm{mW}$.

Measurement of the produced charge current and calculation of the inverse Edelstein length. The inverse Edelstein length $\lambda_{I E E}$ is the figure of merit quantifying the efficiency of the spin to charge current conversion. It has the dimension of a length, as the $3 \mathrm{D}$ spin current $J_{S}^{3 D}$ (in $\mathrm{A} / \mathrm{m}^{2}$ ) is converted into a $2 \mathrm{D}$ charge current $J_{c}^{2 D}$ (in $\mathrm{A} / \mathrm{m}$ ):

$\lambda_{I E E}=\frac{J_{C}^{2 D}}{J_{S}^{3 D}}$

Both $J_{S}^{3 D}$ and $J_{c}^{2 D}$ need to be evaluated to calculate the Inverse Edelstein length. Here we use the method already described in previous works (for example on LAO/STO ${ }^{26}$ or $\mathrm{HgTe}^{28}$ ).

The produced charge current is simply extracted from the symmetric component of the measured spin signal $V_{\text {sym }}$ :

$J_{C}^{2 D}=\frac{V_{s y m}}{R w}$

where $R$ is the resistance of the sample (measured independently), and $w$ is the sample width $(400 \mu \mathrm{m})$.

Note that here, the produced current $J_{c}^{2 D}$ is used to give the amplitude of the spin signal, as it can be considered as a raw data. In order to have values comparable from measurement to measurement, especially with experiments found in the literature, and as the spin signal varies linearly with the square of the excitation field $\mu_{0} h_{r f}$, the current production has to be normalized. Thus, the produced current given in the main text is actually $J_{c}^{2 D} /\left(\mu_{0} h_{r f}\right)^{2}$, in $A . m T^{-2} m^{-1}$. The radiofrequency field for a given measurement is measured using the cavity conversion factor. 
The spin current is extracted using the spin pumping theory firstly developed by Tserkovnyak, Brataas $^{27,34}$ and coworkers, and then by several other groups ${ }^{35,36}$. The spin current injected at the ferromagnetic resonance can be obtained by measuring some of the magnetic properties of the ferromagnetic layer, and by calculating the effective spin mixing conductance:

$G_{e f f}^{\uparrow \downarrow}=\frac{4 \pi M_{s} t_{F M}}{g \mu_{B}}\left(\alpha-\alpha_{r e f}\right)$

where $\mu_{B}$ is the Bohr magneton, $t_{F M}$ the thickness of the ferromagnetic material ( $20 \mathrm{~nm}$ here), $M_{s}$ the saturation magnetization of the Permalloy thin film, $g$ its g-factor, $\alpha$ its Gilbert damping, and $\alpha_{r e f}$ the Gilbert damping of a Permalloy thin film without spin-sink (here Permalloy on native Si). All these values are extracted from independent FMR measurements, using either broadbandFMR or out-of-plane angular dependence measurements.

Then, using the expression of the spin mixing conductance we can obtain the injected spin current:

$J_{S}^{3 D}=\frac{G_{e f f}^{\uparrow \downarrow} \gamma^{2} \hbar \mu_{0} h_{r f}^{2}}{8 \pi \alpha^{2}}\left[\frac{4 \pi M_{S} \gamma+\sqrt{\left(4 \pi M_{S} \gamma\right)^{2}+4 \omega^{2}}}{\left(4 \pi M_{S} \gamma\right)^{2}+4 \omega^{2}}\right] \frac{2 e}{\hbar}$

Where $\gamma$ is the gyromagnetic ratio, $\omega$ the angular frequency, $e$ the elementary charge and $\hbar$ the reduced Planck constant. The inverse Edelstein length can then be obtained by combining equations (1), (2) and (4).

Reproducibility of the gate voltage dependence of the spin-charge conversion. We have performed spin pumping measurements on different samples of $\mathrm{NiFe}(20 \mathrm{~nm}) / \mathrm{Al}(0.9 \mathrm{~nm}) / / \mathrm{STO}$ at $7 \mathrm{~K}$, cf. Extended Data Figure 1. Sample 1 is taken from a first batch, whereas samples 2 and 3 are two different samples of the same second batch. The results shown in the main text have been measured on Sample 3. After thin film deposition on STO substrates the samples were all thinned down to the same thickness $(250 \pm 20 \mu \mathrm{m})$. As can be seen on the Extended Data Figure 1 , for these three samples similar gate voltage dependences have been obtained, with a hysteretic behavior, a positive or negative remanent spin-signal at $V_{\text {gate }}=0 \mathrm{~V}$, and large conversion efficiencies. The obtained inverse Edelstein lengths $\lambda_{\text {IEE }}$ are above $40 \mathrm{~nm}$ in all three cases, and up to $60 \mathrm{~nm}$ in the case of sample 3. The error bars are mostly due to the uncertainty on the effective spin mixing conductance. The main results presented in the text are thus reproducible, even though the samples have been thinned down using mechanical polishing. 
We have also performed several cool-downs on the same sample. After performing a first cooldown and some gate dependence measurements at low temperature, it is possible to recover the initial state by heating up the sample at room temperature. As can be seen in Extended Data Figure 2 (measured on sample 2), the remanent ferroelectric state is lost after heating, but when going back to $7 \mathrm{~K}$ the sample recovers the initial state, with a lower and positive spin signal. This is consistent with our observation of a voltage-induced ferroelectricity at low temperature. After heating at room temperature, an initialization loop [+200 V; -200 V; +200 V] performed at low temperature allows retrieving the hysteretic behavior and the remanence of the polarisation.

Time stability of the remanent state. In the main text we show that a $\pm 200 \mathrm{~V}$ gate voltage application at $7 \mathrm{~K}$ allows controlling the spin-charge conversion in a remanent way. To demonstrate the non-volatility associated to this remanence, we performed spin pumping measurements hours after applying a gate voltage of either $+200 \mathrm{~V}$ or $-200 \mathrm{~V}$ during $500 \mathrm{~ms}$. As seen in Extended Data Figure 3, the produced normalized current is preserved, remaining unmodified after several hours.

Electric polarisation measurements. In these experiments, a triangular waveform was applied at a frequency of $1 \mathrm{kHz}$ across the STO, between the 2DEG and a bottom electrode of Ti/Au, and the current I was measured in real time. Integrating the current with time and normalizing by the sample area yields the polarisation.

Magnetotransport. Low temperature electrical transport measurements were performed on the thinned samples bonded by Al wires in the van der Pauw configuration using a standard AC lockin technique $\left(I_{A C}=200 \mathrm{nA}, f_{A C}=77.03 \mathrm{~Hz}\right)$ in a Quantum Design Dynacool cryostat at a temperature of $2 \mathrm{~K}$ and magnetic fields between $-9 \mathrm{~T}$ and $9 \mathrm{~T}$ for the Hall resistance study. Prior to any backgate voltage data was recorded, the samples were subjected to a so-called forming step ${ }^{30}$ at $2 \mathrm{~K}$, where the back-gate voltage were cycled several times $(>2)$ between the gate voltage extremes of the particular gate-voltage interval to ensure no irreversible changes would occur in the interface system upon application of the back-gate voltage in the actual experiment. Note that this low temperature forming step was repeated following all occasions the sample was brought above $105 \mathrm{~K}$. Moreover, at each new cooldown, the samples were always cooled with the backgate electrostatically grounded. 
R-V loops measured on $\mathrm{NiFe} / \mathrm{Al} / \mathrm{SrTiO}_{3}$ samples. Extended Data Figure 3 shows $\mathrm{R}-\mathrm{V}$ loops measured on the NiFe/AI/STO sample used for spin-pumping. The $R-V$ and $J_{c}-V$ loops have rather similar shapes, indicating a similar origin for both hysteresis. The observed two-probe resistance variation of $\sim 0.27 \Omega$ in this $0.4 \mathrm{~mm} \times 2.4 \mathrm{~mm} \mathrm{NiFe}(20 \mathrm{~nm}) /$ AlOx//STO sample is compatible with the $R-V$ for an AlOx//STO sample shown in Fig. $4 \mathrm{~b}$. The room-temperature sheet resistance of the $\mathrm{NiFe}(20 \mathrm{~nm}) / \mathrm{AlOx} / /$ STO sample is roughly that of the NiFe layer, and equal to $9 \Omega$. In Fig. 4, gating results in a change of the $2 \mathrm{DEG}$ sheet resistance from about $1.7 \mathrm{k} \Omega$ to $23.5 \mathrm{k} \Omega$. In a simple parallel model of the $\mathrm{NiFe}(20 \mathrm{~nm}) / \mathrm{AlOx} / / \mathrm{STO}$ sample (in which current flows in parallel in the NiFe and the 2DEG ), gating should thus result in a sheet resistance change of

$\Delta R=\left(\frac{R_{S}^{2 D E G} R_{S}^{N i F e}}{R_{S}^{2 D E G}+R_{S}^{N i F e}}\right)_{V G-}-\left(\frac{R_{S}^{2 D E G} R_{S}^{N i F e}}{R_{S}^{2 D E G}+R_{S}^{N i F e}}\right)_{V G+}=\frac{23500 \times 9}{23500+9}-\frac{1700 \times 9}{1700+9}=0.044 \Omega$

corresponding to an expected two-probe resistance change of $0.26 \Omega$, in excellent with the observed change of $0.27 \Omega$.

The shape of the $P-V$ and $R-V$ loops of Fig. 4 is different from that of the $J_{C}-V$ and $R-V$ data of Extended Data Fig. 3. One reason is that the spin-pumping experiments were performed on a $\mathrm{Al} / / \mathrm{STO}$ sample covered with a NiFe layer to perform the spin injection whereas the $R-E$ and $P-E$ loops were performed on $\mathrm{Al} / / \mathrm{STO}$ samples without NiFe thus different electrostatic geometry. Additionally, the sample dimensions are also different for the two sets of experiments. In the SPFMR experiments, the STO thickness is $250 \mu \mathrm{m}$, and the lateral size is $0.4 \mathrm{~mm} \times 2.4 \mathrm{~mm}$. For the $R-E$ and $P$-E loop experiments, the STO thickness is $200 \mu \mathrm{m}$ and the lateral size is of $5 \mathrm{~mm} \times 5 \mathrm{~mm}$. Finally, the SP-FMR samples are cut from plain samples, which could induce defects modifying the coercivity. We believe the observed discrepancy between loops to arise primarily from these abovementioned differences.

\section{ACKNOWLEDGEMENTS}

The authors thank M. Cazayous, B. Dkhil, M. Maglione, S. Gambarelli and V. Maurel for useful discussions. This work received support from the ERC Consolidator grant no. 615759 "MINT", the ERC Advanced grant n 833973 "FRESCO", the QUANTERA project "QUANTOX", the French 
Research Agency (ANR) as part of the "Investissement d'Avenir" program (LABEX NanoSaclay, ref ANR-10-LABX-0035) through project "AXION" and the Laboratoire d'Excellence LANEF (ANR-10LABX-51-01) and ANR project OISO (ANR-17-CE24-0026-03). F. Trier acknowledges support by research grant VKR023371 (SPINOX) from VILLUM FONDEN.

\section{Author CONTRibutions}

JPA, PN, LV and MB designed the experiment. JPA, LV and MB supervised the study. DCV, LMVA and JB prepared the samples. PN performed the spin-charge conversion experiments with JPA and LV. JB, SF and MB performed the polarisation measurements with the help of VG and FT. FT and JB performed the transport experiments and analyzed them with $M B$ and $A B$. MB and JPA wrote the paper with inputs from all authors.

\section{COMPETING INTERESTS}

The authors declare no competing interests.

\section{DATA AND MATERIALS AVAILABILITY}

The data that support the findings of this study are available from the corresponding authors upon reasonable request. 


\section{REFERENCES}

1. Jones, N. The information factories. Nature 561, 163-167 (2018).

2. Manipatruni, S., Nikonov, D. E. \& Young, I. A. Beyond CMOS computing with spin and polarization. Nat. Phys. 14, 338-343 (2018).

3. Žutić, I., Fabian, J. \& Das Sarma, S. Spintronics: Fundamentals and applications. Rev. Mod. Phys. 76, 323-410 (2004).

4. Slonczewski, J. C. Current-driven excitation of magnetic multilayers. J. Magn. Magn. Mater. 159, L1-L7 (1996).

5. Heron, J. T. et al. Deterministic switching of ferromagnetism at room temperature using an electric field. Nature 516, 370-373 (2014).

6. Gränicher, H. Induzierte Ferroelektricität von $\mathrm{SrTiO}_{3}$ bei sehr tiefen Temperaturen und über die Kälteerzeugung durch adiabatische Entpolarisierung. Helvetica Phys. Acta 29, 210-212 (1956).

7. Hemberger, J., Lunkenheimer, P., Viana, R., Böhmer, R. \& Loidl, A. Electric-field-dependent dielectric constant and nonlinear susceptibility in $\mathrm{SrTiO}_{3}$. Phys. Rev. B 52, 13159-13162 (1995).

8. Sidoruk, J. et al. Quantitative determination of domain distribution in $\mathrm{SrTiO}_{3}$ - competing effects of applied electric field and mechanical stress. J. Phys. Condens. Matter 22, 235903 (2010).

9. Manaka, H., Nozaki, H. \& Miura, Y. Microscopic Observation of Ferroelectric Domains in $\mathrm{SrTiO}_{3}$ Using Birefringence Imaging Techniques under High Electric Fields. J. Phys. Soc. Jpn. 86, 114702 (2017). 
10. Caviglia, A. D. et al. Tunable Rashba Spin-Orbit Interaction at Oxide Interfaces. Phys. Rev. Lett. 104, 126803 (2010).

11. Ohtomo, A. \& Hwang, H. Y. A high-mobility electron gas at the $\mathrm{LaAlO}_{3} / \mathrm{SrTiO}_{3}$ heterointerface. Nature 427, 423-426 (2004).

12. Soumyanarayanan, A., Reyren, N., Fert, A. \& Panagopoulos, C. Emergent phenomena induced by spin-orbit coupling at surfaces and interfaces. Nature 539, 509-517 (2016).

13. Edelstein, V. M. Spin polarization of conduction electrons induced by electric current in twodimensional asymmetric electron systems. Solid State Commun. 73, 233-235 (1990).

14. Kondou, K. et al. Fermi-level-dependent charge-to-spin current conversion by Dirac surface states of topological insulators. Nat. Phys. 12, 1027-1031 (2016).

15. Hoffmann, A. Spin Hall Effects in Metals. IEEE Trans. Magn. 49, 5172-5193 (2013).

16. Bychkov, Y. A. \& Rashba, E. I. Properties of a 2D electron gas with lifted spectral degeneracy. JETP Lett. 39, 78-81 (1984).

17. Sánchez, J. C. R. et al. Spin-to-charge conversion using Rashba coupling at the interface between non-magnetic materials. Nat. Commun. 4, (2013).

18. Picozzi, S. Ferroelectric Rashba semiconductors as a novel class of multifunctional materials. Front. Phys. 2, (2014).

19. Rinaldi, C. et al. Ferroelectric Control of the Spin Texture in GeTe. Nano Lett. 18, 2751-2758 (2018).

20. Kolobov, A. V. et al. Ferroelectric switching in epitaxial GeTe films. APL Mater. 2, 066101 (2014).

21. Rinaldi, C. et al. Evidence for spin to charge conversion in GeTe(111). APL Mater. 4, (2016). 
22. Bibes, M., Vila, L., Attané, J.-P., Noël, P. \& Vaz, D. C. Dispositif électronique, porte numérique, composant analogique et procédé de génération d’une tension (Patent number FR18 74319).

23. Manipatruni, S. et al. Scalable energy-efficient magnetoelectric spin-orbit logic. Nature 565, 35-42 (2019).

24. Rödel, T. C. et al. Universal Fabrication of 2D Electron Systems in Functional Oxides. Adv. Mater. 28, 1976-1980 (2016).

25. Vaz, D. C. et al. Mapping spin-charge conversion to the band structure in a topological oxide two-dimensional electron gas. Nat. Mater. 18, 1187-1193(2019)

26. Lesne, E. et al. Highly efficient and tunable spin-to-charge conversion through Rashba coupling at oxide interfaces. Nat. Mater. 15, 1261-1266 (2016).

27. Tserkovnyak, Y., Brataas, A. \& Bauer, G. E. W. Enhanced Gilbert Damping in Thin Ferromagnetic Films. Phys. Rev. Lett. 88, 117601 (2002).

28. Noel, P. et al. Highly Efficient Spin-to-Charge Current Conversion in Strained HgTe Surface States Protected by a HgCdTe Layer. Phys. Rev. Lett. 120, 167201 (2018).

29. Caviglia, A. D. et al. Electric field control of the $\mathrm{LaAlO}_{3} / \mathrm{SrTiO}_{3}$ interface ground state. Nature $456,624-627$ (2008).

30. Biscaras, J. et al. Limit of the electrostatic doping in two-dimensional electron gases of $\mathrm{LaXO}_{3}(\mathrm{X}=\mathrm{Al}, \mathrm{Ti}) / \mathrm{SrTiO}_{3}$. Sci. Rep. 4, 6788 (2015).

31. Crassous, A. et al. Nanoscale Electrostatic Manipulation of Magnetic Flux Quanta in Ferroelectric/Superconductor $\mathrm{BiFeO}_{3} / \mathrm{YBa}_{2} \mathrm{Cu}_{3} \mathrm{O}_{7-\delta}$ Heterostructures. Phys. Rev. Lett. 107, 247002 (2011). 
32. Yamada, H. et al. Ferroelectric control of a Mott insulator. Sci. Rep. 3, 2834 (2013).

33. Haeni, J. H. et al. Room-temperature ferroelectricity in strained $\mathrm{SrTiO}_{3}$. Nature 430, 758-761 (2004).

34. Brataas, A., Tserkovnyak, Y., Bauer, G. E. W. \& Halperin, B. I. Spin battery operated by ferromagnetic resonance. Phys. Rev. B 66, 060404 (2002).

35. Costache, M. V., Sladkov, M., Watts, S. M., van der Wal, C. H. \& van Wees, B. J. Electrical Detection of Spin Pumping due to the Precessing Magnetization of a Single Ferromagnet. Phys. Rev. Lett. 97, 216603 (2006).

36. Ando, K. et al. Inverse spin-Hall effect induced by spin pumping in metallic system. J. Appl. Phys. 109, 103913 (2011). 


\section{EXTENDED DATA}

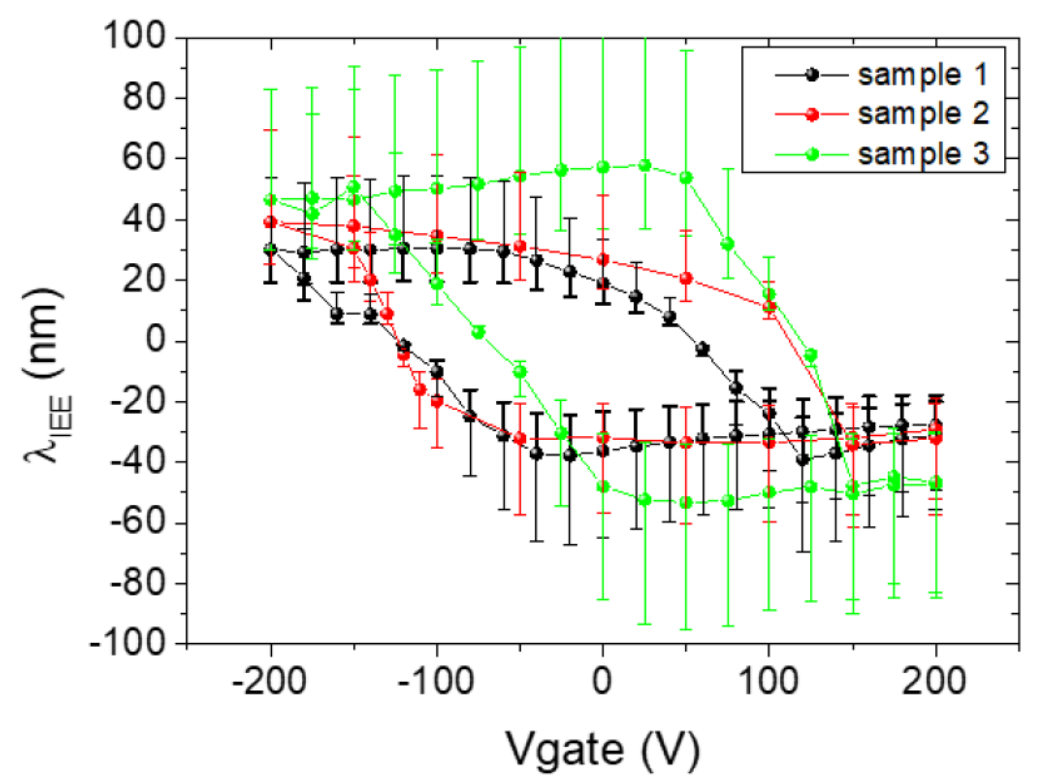

Extended Data Figure 1. Gate voltage dependence of the inverse Edelstein length in three different samples of $\mathrm{NiFe}(20 \mathrm{~nm}) / \mathrm{Al}(0.9 \mathrm{~nm}) / / \mathrm{STO}$. The error bars are due to the small extra damping measured in this system. The estimated effective spin mixing conductance $G_{e f f}^{\uparrow \downarrow}$ is ranging from $1.2 \mathrm{~nm}^{-2}$ to $3.2 \mathrm{~nm}^{-2}$ with a mean value of $2.2 \mathrm{~nm}^{-2}$, leading to an injected spin current $J_{S}^{3 D}$ ranging from 100 to $240 \mathrm{MA} \cdot \mathrm{m}^{-2} \cdot \mathrm{mT}^{-2}$, with a mean value of $160 \mathrm{MA} \cdot \mathrm{m}^{-2} \cdot \mathrm{mT}^{-2}$ (see Supplementary Information). 


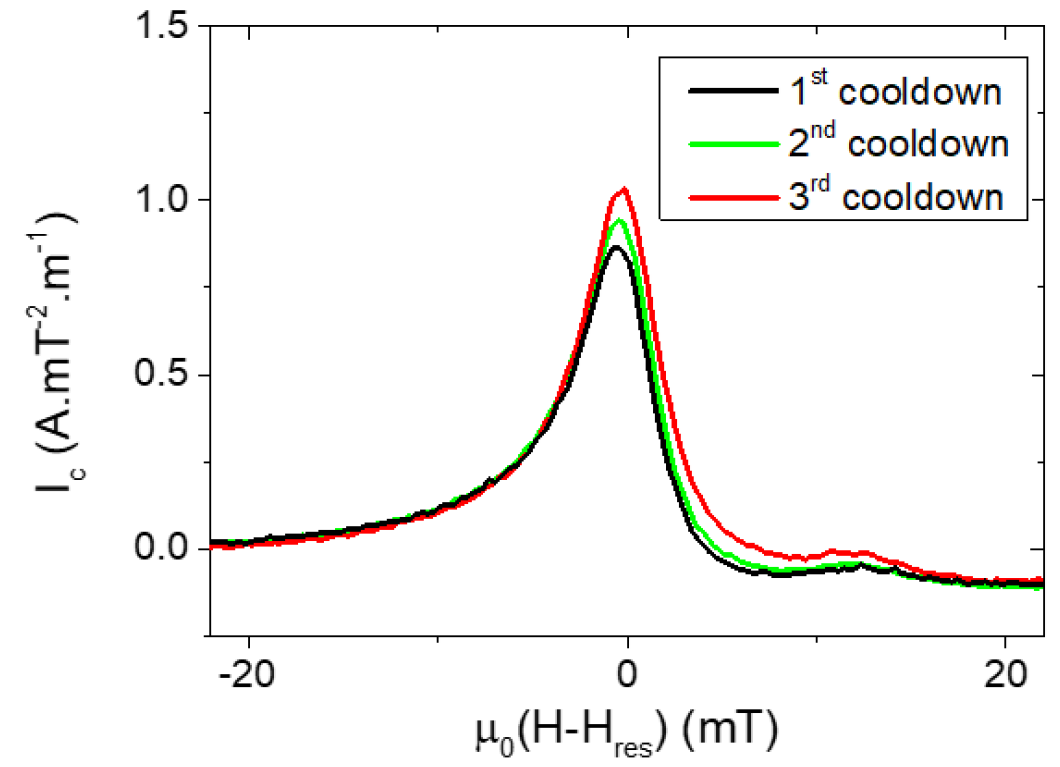

Extended Data Figure 2. Spin pumping signals obtained at 7K on sample 2, for three different cooldowns from room temperature. After each cooldown, the signal was measured before any gate voltage application. 


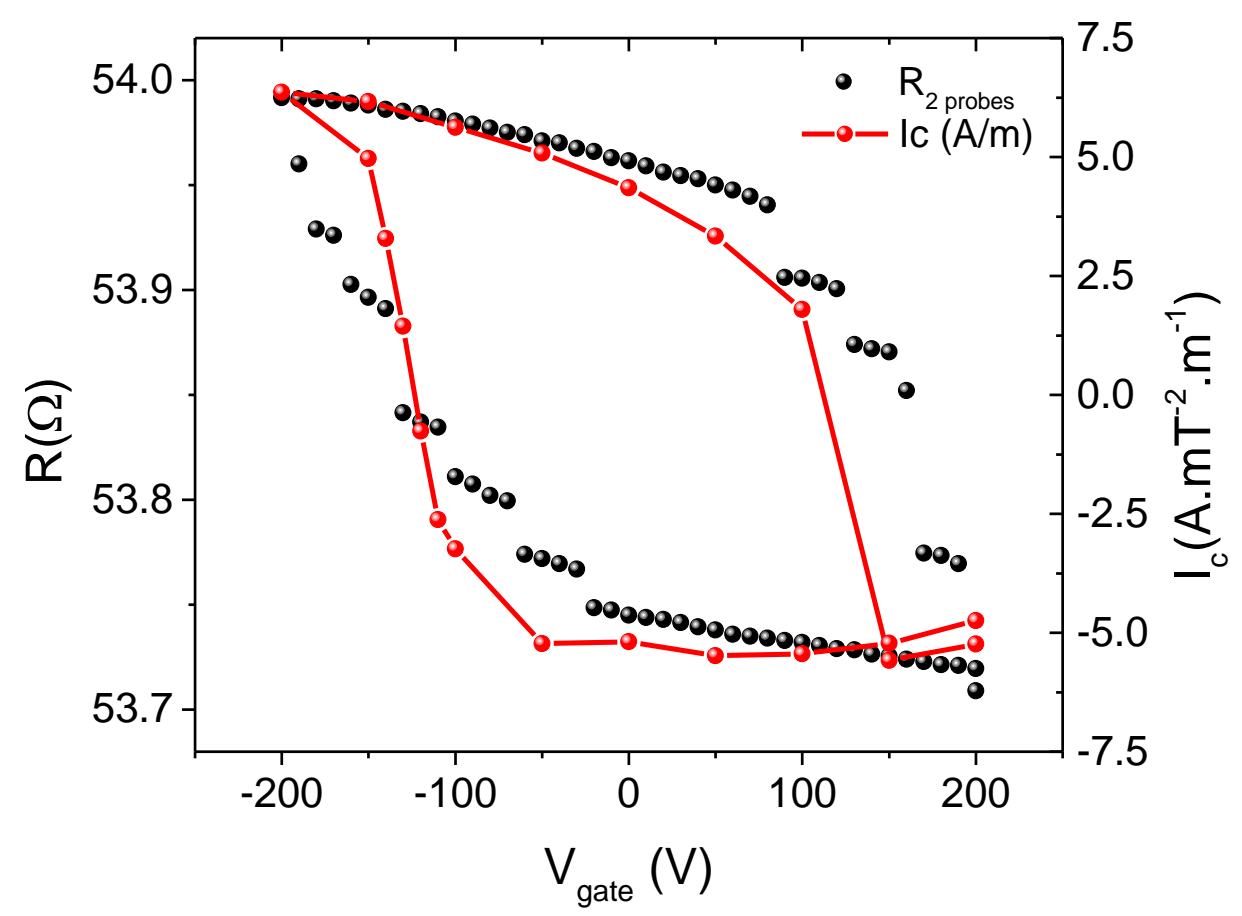

Extended Data Figure 3: Two-probe resistance of a NiFe/AI/STO sample, measured in the spin pumping setup as a function of the back-gate voltage (in black), and normalized charge current production measured by spin pumping (in red). 


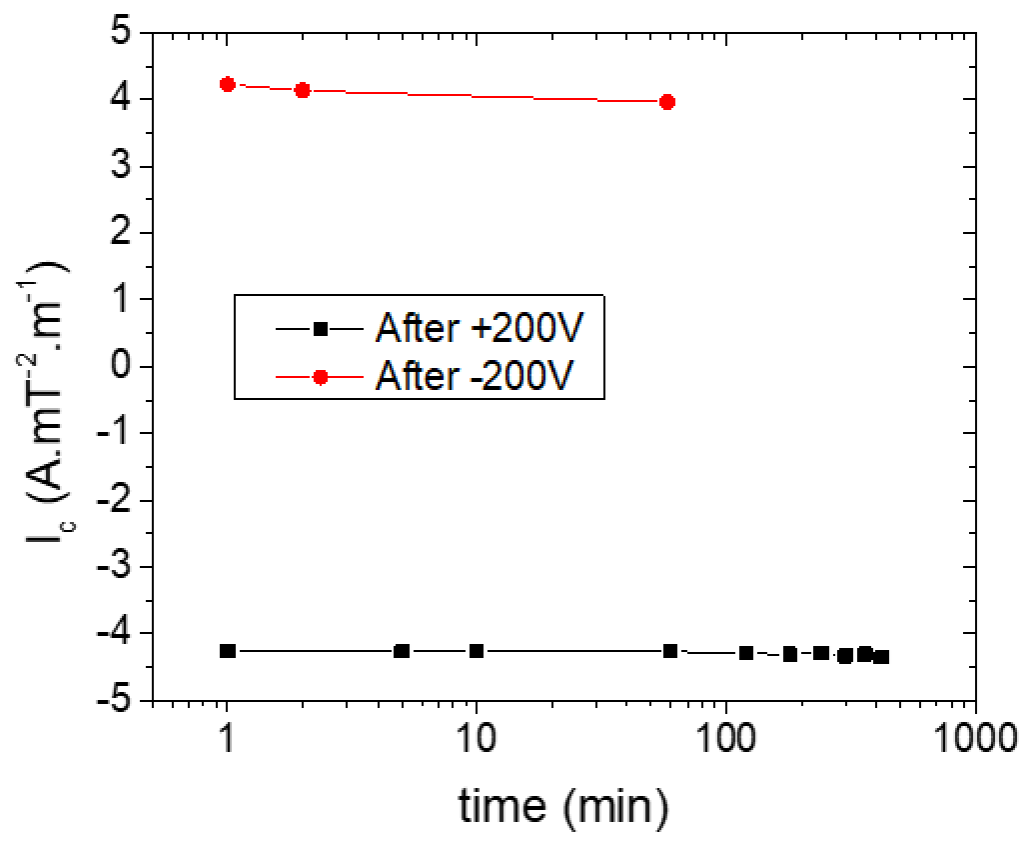

Extended Data Figure 4. Dependence of the produced current with the time spent after application of a positive (black) or negative (red) gate voltage. The measurements were performed at 7K on sample 1. 